\title{
Cellular Network Non-Line-of-Sight Reflector Localisation Based on Synthetic Aperture Radar Methods
}

\author{
Zoran Sjanic, Fredrik Gunnarsson, Carsten Frtische and Fredrik Gustafsson
}

\section{Linköping University Post Print}

\section{Tweet}

N.B.: When citing this work, cite the original article.

C2014 IEEE. Personal use of this material is permitted. However, permission to reprint/republish this material for advertising or promotional purposes or for creating new collective works for resale or redistribution to servers or lists, or to reuse any copyrighted component of this work in other works must be obtained from the IEEE.

Zoran Sjanic, Fredrik Gunnarsson, Carsten Frtische and Fredrik Gustafsson, Cellular Network Non-Line-of-Sight Reflector Localisation Based on Synthetic Aperture Radar Methods, 2014, IEEE Transactions on Antennas and Propagation, (62), 4, 2284-2287.

http://dx.doi.org/10.1109/TAP.2014.2300531

Postprint available at: Linköping University Electronic Press

http://urn.kb.se/resolve?urn=urn:nbn:se:liu:diva-97279 


\section{Cellular Network Non-Line-of-Sight Reflector Localisation Based on Synthetic Aperture Radar Methods}

\author{
Zoran Sjanic, Fredrik Gunnarsson Member IEEE, Carsten \\ Fritsche Member IEEE and Fredrik \\ Gustafsson Fellow IEEE
}

\begin{abstract}
The dependence of radio signal propagation on the environment is well known, and both statistical and deterministic methods have been presented in the literature. Such methods are either based on randomised or actual reflectors of radio signals. In this work, we instead aim at estimating the location of the reflectors based on geo-localised radio channel impulse response measurements using methods from synthetic aperture radar (SAR). Radio channel measurements from 3GPP E-UTRAN have been used to verify the usefulness of the proposed approach. The obtained images show that the estimated reflectors are well-correlated with the aerial map of the environment. Also, trajectory segment contributions to different reflectors have been estimated with promising results.
\end{abstract}

Index Terms - Synthetic Aperture Radar, Radar Imaging, Frequency Divison Multiplexing

\section{INTRODUCTION}

Radio signal propagation significantly depends on the environment through which the signal propagates. Free space propagation is different from line-of-sight (LOS) propagation close to the ground, which in turn is different from non-line-ofsight (NLOS) propagation where obstacles and reflectors have a significant impact on the propagation properties. Modeling radio signal propagation properties based on environment characteristics is therefore important to properly plan and analyse terrestrial wireless communication systems.

Numerous activities are focusing on propagation channel modeling featuring NLOS propagation, and the efforts can be separated into statistical models and ray-tracing models. The former can be motivated by theoretical modeling, or designed as parametric models that can be tuned and validated based on empirical data. The surveys [1], [2] cover statistical models well. One key statistical model example is the 3GPP Spatial Channel Model (SCM) [3], which has been empirically validated [4]. In brief, the model describes random scatterers that reflect incident radio waves toward the radio receiver. Raytracing models [1], [5] on the other hand, aim at modeling the properties of the physical multi-path propagation channel, including knowledge about the location of scatterers and

Z. Sjanic is with the Division of Automatic Control, Department of Electrical Engineering, Linköping University, SE-581 83 Linköping, Sweden (e-mail: zoran@isy.liu.se)

F. Gunnarsson is with the Division of Automatic Control, Department of Electrical Engineering, Linköping University, SE-581 83 Linköping, Sweden and with Ericsson Research, Linköping, Sweden (e-mail: fred@isy.liu.se, fredrik.gunnarsson@ericsson.com)

C. Fritsche is with IFEN GmbH, Alte Gruber Str. 6, 85586 Poing, Germany (e-mail: carsten@isy.liu.se)

F. Gustafsson is with the Division of Automatic Control, Department of Electrical Engineering, Linköping University, SE-581 83 Linköping, Sweden (e-mail: fredrik@isy.liu.se)

The authors gratefully acknowledge funding from the Vinnova Industry Excellence Center LINK-SIC and the SSF project Cooperative Localization. reflectors. Such information can be derived from 3-D building databases but will still be associated with some uncertainty.

In order to model the NLOS propagation in a specific area, one either needs to tune the parameters of the statistical model so that it generates reflectors representative for the area, or to analyse building data to determine the reflectors and occlusions deterministically. In this paper, we take an intermediate approach by using geo-localised impulse response measurements to estimate the reflector locations. Furthermore, the ambition is also to estimate where in an area a particular reflector is active. Since the number of reflectors is unknown, a non-parametric method must be applied to get an initial map of the reflector locations that can be refined with some parametric method afterwards. One non-parametric method to get an initial estimate of the map is based on a multistatic Synthetic Aperture Radar (SAR)-like technique, [6], [7], [8].

The paper is organised as follows; Section II describes SAR in general terms, and Section III describes the considered application based on measurements of 3GPP Long Term Evolution. In Section IV, the radio channel measurement campaign is described, while Section V adopts the SAR methods to enable reflector estimation and provides some results. Finally, Section VI concludes the paper.

\section{SAR AND MULTISTATIC SAR}

SAR imaging is based on a platform moving along a scene that is to be imaged. During the movement, the platform transmits radar pulses which hit the scene and return to the platform with a certain time delay proportional to the range to the scene. This returned signal is match filtered and then sampled. Each reflector in the scene will contribute a scattered power and will then be placed in an appropriate range bin. The range $R$ is determined as a product between signal propagation speed (usually speed of light, $c_{0}$ ) and delay time. In this way a single scene transfer function is obtained, denoted $g(R)$. Now this process can be repeated during the platform movement at different time instances $t$, yielding a transfer function $g_{t}(R)$ which can be stored in a two-dimensional array. Basically, this raw data, $g_{t}(R)$, is an example of a real aperture radar or RAR. The resolution in such a radar system is proportional to the radar beamwidth and is usually quite poor. One important thing to notice is that the beamwidth is inversely proportional to the antenna size, i.e. the larger antenna the smaller the beamwidth we can obtain.

The idea behind SAR is to artificially synthesise a large antenna by moving the platform, [6], [9]. One way that this can be done is by a global back-projection method, [10], [11], [12], that can be described in the following way; given the raw (possibly complex valued) data $g_{t}(R)$, we can back-project each radar echo on the image yielding the subimage $I_{t}$ and each reflector will create a circle in each subimage. A total image $I$ can then be created by summing up all the subimages along the synthetic aperture

$$
I=\sum_{t=1}^{N} I_{t}
$$




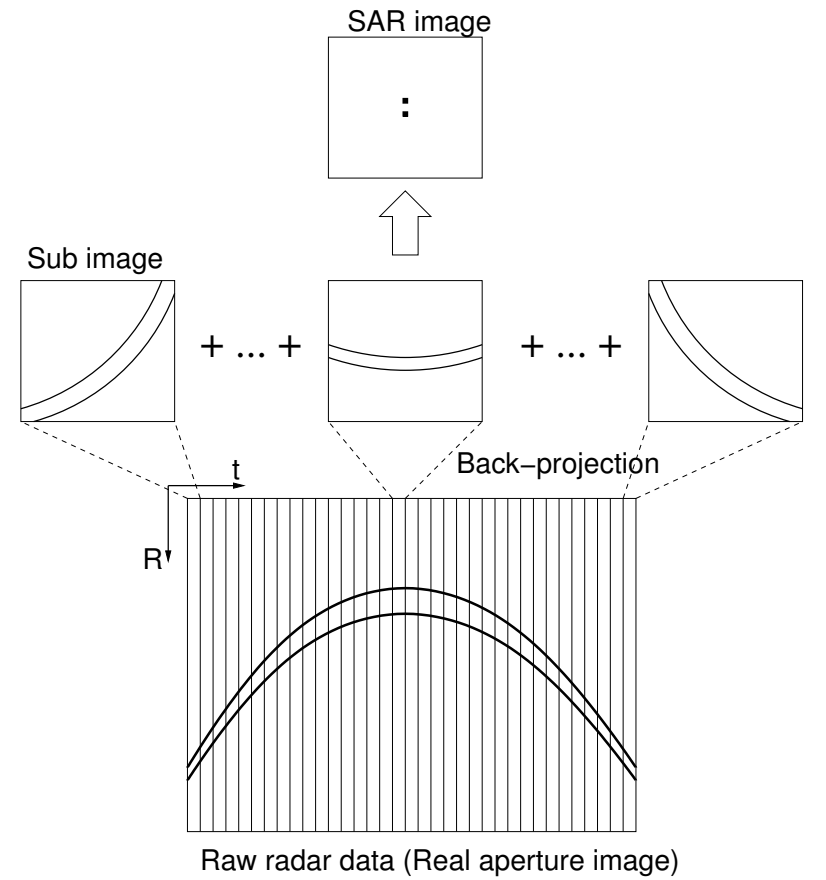

Figure 1: Global back-projection method for creating SAR images. The scene consists of two point targets.

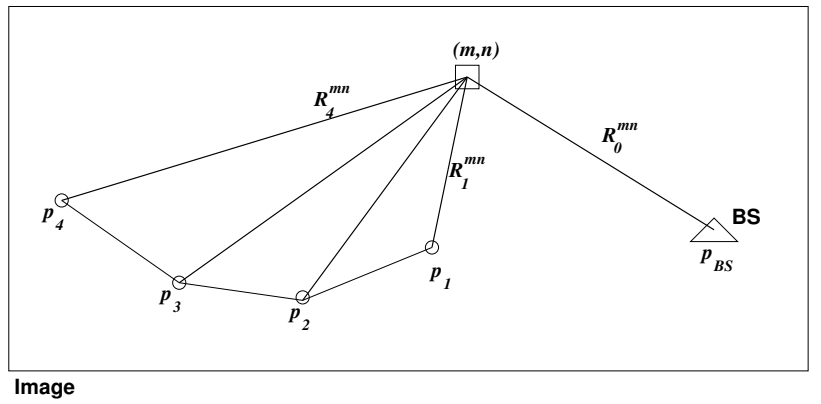

Figure 2: Example of a multistatic SAR geometry in two dimensions.

This method can schematically be described as in Figure 1. Another way of creating the image is to integrate the raw data for each pixel in the resulting image $I_{m n}$ as

$$
\begin{aligned}
I_{m n} & =\sum_{t=1}^{N} g_{t}\left(R_{t}^{m n}\right) \\
R_{t}^{m n} & =\left\|p_{t}-s^{m n}\right\|_{2}
\end{aligned}
$$

where $p_{t}$ is the position of the platform, $s^{m n}$ is the position in the scene which corresponds to the pixel $(m, n)$ and $\|\cdot\|_{2}$ is the usual vector L2-norm. The complexity of this operation is proportional to $\mathcal{O}\left(N M^{2}\right)$ for an $M \times M$ image and $N$ time points. This describes the principle of monostatic SAR in which transmitting and receiving antennas are co-located. The principle can be generalised with transmitting and receiving antennas at different positions to principles of multistatic SAR. Also, the specific case of SAR with one separate transmitting antenna and multiple receiving antennas or antenna positions, is highly relevant.

Figure 2 illustrates a case with one transmitter (or base station) in one position $p_{B S}$ and a receiving antenna at positions $p_{1}$ to $p_{4}$. The only difference to the case in (2) is to add the distance $R_{0}^{m n}$ between the transmitter and the pixel coordinate $(m, n)$ to the total range.

$$
\begin{aligned}
I_{m n} & =\sum_{t=1}^{N} g_{t}\left(R_{0}^{m n}+R_{t}^{m n}\right) \\
R_{0}^{m n} & =\left\|p_{B S}-s^{m n}\right\|_{2} \\
R_{t}^{m n} & =\left\|p_{t}-s^{m n}\right\|_{2}
\end{aligned}
$$

In this case each reflector will describe an ellipse in the image with focal points located in $p_{B S}$ and $p_{t}$. It shall be noted that the integration above can be done with the complex valued raw data (coherent) or with the magnitude of the complex valued data (incoherent).

\section{OFDM SIGNAL AND SAR MODELING}

In this section, the considered OFDMA signals are modeled, and associations to the SAR theory are established.

Even though the methods described next apply to a general radio network, the modeling will adopt 3GPP LTE [13] nomenclature and describe broadcasted signals from base stations based on Orthogonal Frequency Division Multiplexing (OFDM). The transmitted signal consists of coded symbols which can be described as

$$
d_{t}=\sum_{k=0}^{N-1} S_{k} e^{j 2 \pi k \frac{t}{T}}, \quad 0 \leq t<T
$$

where $S_{k}$ are the transmitted symbols, $N$ is the number of symbols and $T$ is the OFDM symbol time and the bandwidth needed is $B=N / T$. This baseband signal is then transformed to a passband signal centered at carrier frequency $f_{c}$

$$
z_{t}=\Re\left\{e^{j 2 \pi f_{c} t} d_{t}\right\}, \quad 0 \leq t<T
$$

where $\Re\{\cdot\}$ denotes the real part of a complex number. The received signal $y_{t}$ should ideally be a scaled (by $A_{0}$ ) and time delayed (by $\tau_{0}$ ) version of the transmitted signal (i.e. the LOS signal)

$$
y_{t}=A_{0} z_{t-\tau_{0}}+e_{t}
$$

where $e_{t}$ denotes some channel noise. However, because of the urban environment and presence of multipath signals, the actual received signal can be written as

$$
y_{t}=\sum_{p=0}^{P} A_{p} z_{t-\tau_{p}}+e_{t}
$$

where the number of multipath reflectors is $P$. In general, the amplitudes $A_{p}$ will be proportional to $\left(R_{t}^{p}\right)^{-\alpha}=\left(c_{0} \tau_{p}\right)^{-\alpha}$, where $\alpha$ is the path loss exponent. However, many amplitudes, including the LOS amplitude, can also be zero, for example if there is an occlusion present. In this paper, we assume that NLOS propagation is due to horizontal reflections. However, it is also possible that it can be due to vertical signal paths, for example via diffraction which means that the signal bends down behind a building, but this is not considered here.

The received signal is usually collected in the frequency domain since the matched filtering can be implemented with 


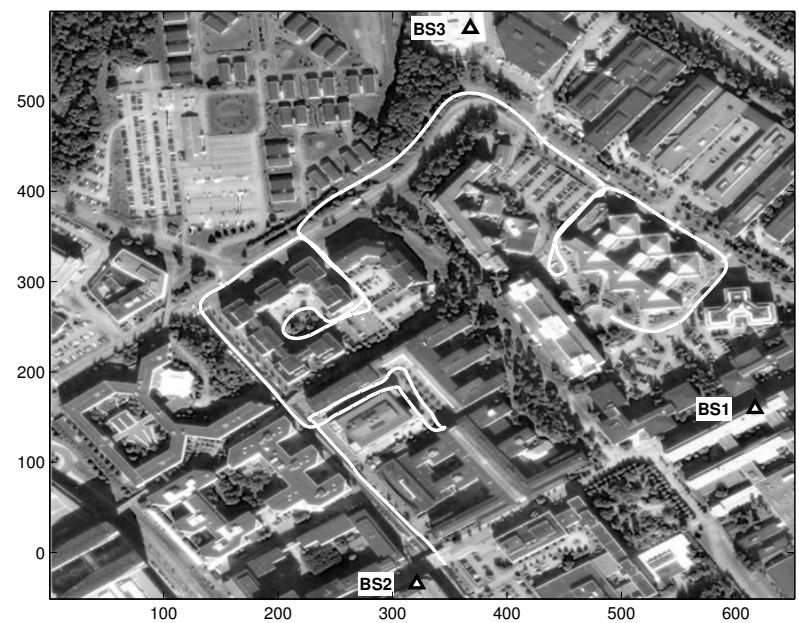

Figure 3: Configurations of the three base stations used in the experiment in Kista, Stockholm. The driven trajectory is in white starting near base station 2 in the lower part of the image.

multiplications instead of convolutions, and the time domain signal is simply obtained as an inverse discrete Fourier transform (IDFT) of the frequency signal, $y_{t}=\mathcal{F}^{-1}\left\{Y_{f}\right\}$. The received signal or impulse response $y_{t}$ can be seen as the scene transfer function for one time instant during the data acquisition, i.e. $y_{t}=g_{t}(R)$ according to the SAR notation.

\section{Radio Channel Measurements}

Radio channel data has been gathered during initial EUTRAN trials in Kista Stockholm. More complete details can be obtained from [14]. The base station configuration was according to Figure 3. At all sites the antennas were mounted a few meters above the average rooftop level which is about $25 \mathrm{~m}$. Three base stations are used which are equipped with commercially available antennas having transmit power of about $35 \mathrm{dBm}$ at the antenna ports. The four receiving antennas were mounted on the rooftop of a van and synchronisation of the transmitting and receiving signal is obtained by rubidium clocks from Stanford Research Systems with Allan standard deviation less than $10^{-12} \mathrm{~s}$, which corresponds to an error in propagation of less than $1 \mathrm{~m}$ during an $8 \mathrm{~min}$ period.

In the actual experiment, the symbols are transmitted over the whole bandwidth $B \approx 20 \mathrm{MHz}$ on the carrier frequency $f_{c}=2.66 \mathrm{GHz}$. The frequency response of the channel is sampled every $\Delta t=51 \mathrm{~ns}$ with the frequency resolution of $\Delta f=45 \mathrm{kHz}$ and saved in a memory with $L=432$ samples. This gives the effective range resolution of $c_{0} /((L-1) \Delta f) \approx$ $15 \mathrm{~m}$. The frequency response is transformed to the spatial domain with the IDFT for each time instant. On top of measuring the base station signals, the Global Positioning System (GPS) based position of the receiver is saved for each time instant. The GPS position data are also synchronised to the signal receiving times with the high accuracy clock. Note also that the error in GPS position is normally less than the range resolution which makes the evaluation of the method feasible.

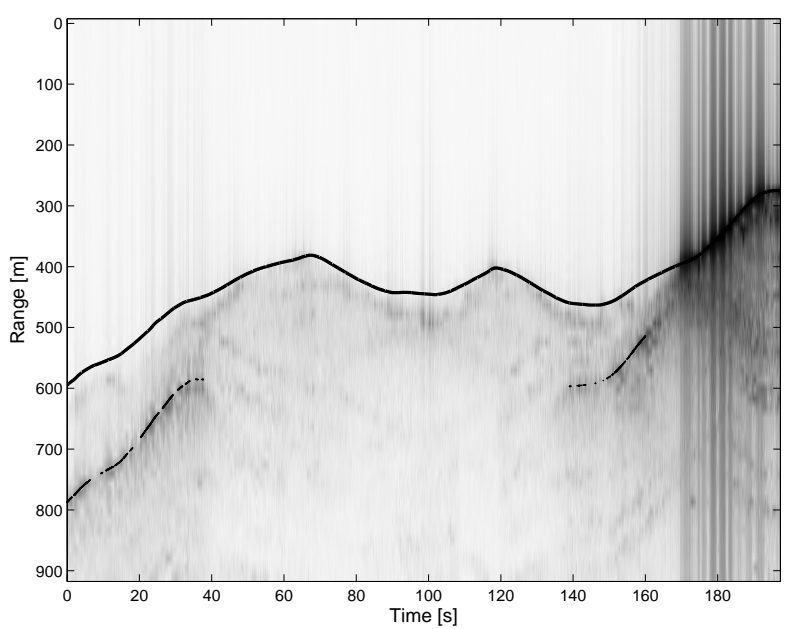

Figure 4: Raw data (logarithm of the magnitude of the impulse response) from one base station. Solid black line is the range to the base station 3 (LOS range) based on the GPS trajectory. Non-continuous line is the NLOS range to the point $(100,300)$ in Figure 6.

An example of the impulse response data collected from one base station (BS3) and averaged over all 4 receiving antennas is depicted in Figure 4. Also the LOS range to the base station is plotted with the solid black line. It can be seen in this figure that during certain times obvious occlusions happen (i.e., no LOS propagation path is present), for example in the time interval between ca. 0 to $170 \mathrm{~s}$. This time interval corresponds to the parts of the trajectory located at the farthest end from base station 3 between coordinates ca. $(350,0)$ and $(150,280)$ in Figure 3, where high buildings are a probable cause for the occlusions. The non-continuous line segments mark the parts of the impulse response with the most evident non-line of sight propagation. The line segments are further discussed in Section V.

\section{REsUlts}

The resulting image of the estimated reflectors can be seen in Figure 5. The estimated image, in grayscale, of the reflectors for all three base stations is overlaid on the image of the Kista area in Stockholm. This image can be interpreted as a likelihood function for the presence of the reflectors. To enhance the visibility, the level curves are also drawn. The trajectory used for the estimation is plotted as a thick black line, and three base stations, denoted BS1, BS2 and BS3, are shown as black triangles. The estimated reflectors seem to be well correlated with the aerial map of the environment, although their resolution is somewhat low. This is a consequence of the SAR processing where many time points (full aperture) are needed in order to obtain the full image resolution, see Section II. In many cases here, the reflectors were visible only for short periods of time causing the low resolution in the image. Another unmodeled aspect is NLOS effects due to vertical signal diffraction, which also may contribute to the reduced resolution.

The reflections can be further analysed by identifying parts of the trajectory where the identified reflector has been active. 


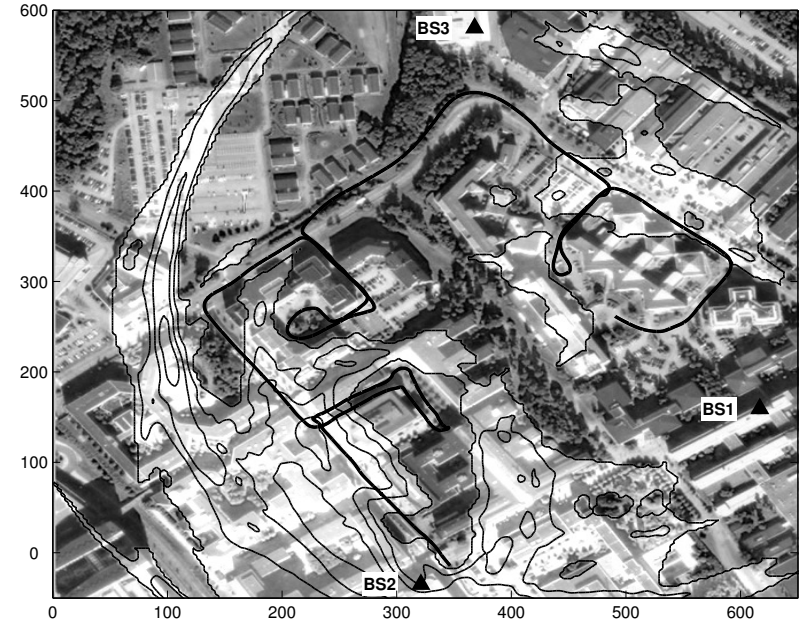

Figure 5: Image of the reflectors estimated from all three base stations in light gray together with level curves overlaid on the test area image. The trajectory is drawn as a thick black line and base station positions are represented with black triangles.



Figure 6: Identified reflector marked with a white square, together with the parts of the trajectory (in black) that are associated to this reflector.

Figure 6 illustrates such an analysis with an identified reflector marked as a white square at coordinate $(100,300)$ in the image. Parts of the trajectory that contribute to that reflector point seen from base station BS3 are highlighted in black. The result is natural, since BS3 is not directly visible from that part of the trajectory due to the occlusion from the buildings. Instead, signals from BS3 propagate via the identified reflector to this part of the trajectory. These parts of the trajectory are also marked with corresponding NLOS range, via the identified reflector in Figure 4. Clearly, these markings coincide with the impulse response and explain the NLOS component. Note that the interruption and the NLOS component in Figure 4 is due to that the van leaves the street with the occlusion for a detour, and when the van returns to the street with the occlusion, the NLOS component is back. Beyond doubt, it is the identified reflector that is active in the NLOS propagation during these segments.

\section{CONCLUSIONS AND Future WORK}

In this paper we devise a method for estimating a map of NLOS reflectors for a mobile radio network based on multistatic SAR imaging. We apply the back-projection principle for the image creation which is a well known method from computerised tomography and also conventional SAR imaging. The obtained images give promising results where the reflectors are well-correlated with the large buildings detectable in the aerial map of the environment. Also, the possibility to extract which part of the trajectory contributed to different reflectors and from which base station is added. In this way a map where dominating reflectors are present can be built up.

The applicability of the estimated reflectors is still unexploited. One possible application area is to consider the estimated reflectors and occlusions in position estimation, [15].

\section{ACKNOWLEDGEMENT}

The evaluation data from the radio channel measurements has been kindly provided by Jonas Medbo, Ericsson.

\section{REFERENCES}

[1] M. A. Jensen and J. W. Wallace, "A Review of Antennas and Propagation for MIMO Wireless Communications," IEEE Trans. on Antennas and Propagation, vol. 52, no. 11, pp. 2810-2824, Nov. 2004.

[2] P. Almers, E. Bonek, A. Burr, N. Czink, M. Debbah, V. Degli-Esposti, H. Hofstetter, P. Kyosti, D. Laurenson, G. Matz, A. Molisch, C. Oestges, and H. Ozcelik, "Survey of Channel and Radio Propagation Models for Wireless MIMO Systems," EURASIP Journal on Wireless Communications and Networking, vol. 2007, no. 1, pp. 1-19, 2007.

[3] 3GPP, "Spatial channel model for Multiple Input Multiple Output (MIMO) simulations, (TS 25.996)."

[4] J. Medbo, M. Riback, and J.-E. Berg, "Validation of 3GPP spatial channel model including WINNER wideband extension using measurements," in In Proc. IEEE Vehicular Technology Conference, 2006.

[5] T. Fügen, J. Maurer, T. Kayser, and W. Wiesbeck, "Capability of 3-D Ray Tracing for Defining Parameter Sets for the Specification of Future Mobile Communications Systems," IEEE Transactions on Antennas and Propagation, vol. 54, no. 11, pp. 3127-3137, Nov. 2006.

[6] L. J. Cutrona, W. E. Vivian, E. N. Leith, and G. O. Hall, "A HighResolution Radar Combat-Surveillance System," IRE Transactions on Military Electronics, vol. MIL-5, no. 2, pp. 127-131, April 1961.

[7] N. Willis, Bistatic Radar, 2nd ed. SciTech, 2007.

[8] V. Krishnan, J. Swoboda, C. Yarman, and B. Yazici, "Multistatic Synthetic Aperture Radar Image Formation," Image Processing, IEEE Transactions on, vol. 19, no. 5, pp. 1290-1306, 2010.

[9] C. Oliver and S. Quegan, Understanding Synthetic Aperture Radar Images, ser. The SciTech Radar and Defense Series. SciTech, 2004.

[10] F. Natterer, The Mathematics of Computerised Tomography. New York: Wiley, 1986.

[11] L. E. Andersson, "On the Determination of a Function from Spherical Averages," SIAM Journal on Mathematical Analysis, vol. 19, no. 1, pp. 214-232, 1988.

[12] J. A. Fawcett, "Inversion of N-Dimensional Spherical Averages," SIAM Journal on Applied Mathematics, vol. 45, no. 2, pp. 336-341, 1985.

[13] E. Dahlman, S. Parkvall, and J. Sköld, 4G LTE/LTE-Advanced for Mobile Broadband, ser. Academic Press. Academic Press, 2011.

[14] J. Medbo, Y. Jading, I. Siomina, and J. Furuskog, "Propagation channel impact on LTE positioning accuracy: A study based on real measurements of observed time difference of arrival," in Personal, Indoor and Mobile Radio Communications, 2009 IEEE International Symposium on, 2009, pp. 2213-2217.

[15] F. Gustafsson and F. Gunnarsson, "Mobile positioning using wireless networks: possibilities and fundamental limitations based on available wireless network measurements," Signal Processing Magazine, IEEE, vol. 22, no. 4, pp. 41-53, 2005. 